zu erklären, warum sie ein Erbscheinsverfahren benötigen und im Anschluss hieran noch bei einem anderen Gericht die Erbenfeststellung vornehmen müssen. So wie das große Familiengericht eingeführt wurde, ist sicherlich auch im Erbrecht Klarheit und Vereinfachung durch ein großes Nachlassgericht herbeizuführen.

\section{Fazit}

Auch der letzte djt 2010 in Berlin hat das Thema unter dem Motto „Ist unser Erbrecht noch zeitgemäß?“ aufgegriffen.
Die djb-Kommission wird sich dem Thema stellen und die entsprechenden frauenpolitischen Zusammenhänge herausarbeiten.

Anpassungen müssen auch schon aufgrund der Entwicklungen auf europäischer Ebene erfolgen.

Noch gehören die jetzt Versterbenden nicht der Scheidungsgeneration an. Es ist jedoch an der Zeit, weiter in die Diskussion einzutreten und das Erbrecht vorausschauend den Anforderungen der sich geänderten und noch ändernden Gesellschaft anzupassen.

\title{
Die Reform des Unterhaltsrechts - eine unendliche Geschichte für die (geschiedene) Ehefrau?
}

\section{Brigitte Meyer-Wehage \\ Mitglied der Kommission Zivil-, Familien- und Erbrecht, Recht an- derer Lebensgemeinschaften des djb; Richterin am AG, Oldenburg}

Das Unterhaltsrechtsänderungsgesetz vom 21. Dezember 2007, welches zum 1. Januar 2008 in Kraft getreten ist, führt mit seinen Neuerungen - bezogen auf den nachehelichen Unterhalt - in der Praxis zu „echten Streitfällen“. 1

Ein kurzer Rückblick: Die Diskussion um die Reform des Unterhaltsrechts wurde durch die Entscheidung des Bundesverfassungsgerichts vom 28. Februar $2007^{2}$ in eine andere Richtung gelenkt. Denn das höchste deutsche Gericht hatte dem Gesetzgeber in die Agenda geschrieben, dass es gegen $\mathrm{Ar}-$ tikel 6 Absatz 5 GG verstoße, die Dauer des Unterhaltsanspruchs eines Kindes daran festzumachen, ob es ehelich oder nichtehelich geboren ist. Zur Erinnerung: Der Unterhalt gemäß \ 1570 BGB war vor der Reform von dem sogenannten „Altersphasenmodell“ geprägt, während $\mathbb{} 16151$ Absatz 2 Satz 3 BGB alte Fassung der Mutter Unterhalt grundsätzlich nur bis zum vollendeten dritten Lebensjahr des Kindes zubilligte.

Der Gesetzgeber hat - in Umsetzung der Entscheidung - alsdann einen auf drei Jahre befristeten „Basisunterhalt“ eingeführt, der aus Gründen der Billigkeit verlängert werden kann. ${ }^{3}$

Dies war der erste tiefe Einschnitt in das bisherige „System" gefolgt von dem nun kodifizierten Grundsatz der Eigenverantwortung. ${ }^{4}$

\section{Entwicklung der Rechtsprechung}

\section{Betreuungsunterhalt}

Mit Urteil vom 16. Juli $2008^{5}$ äußerte sich der Bundesgerichtshof (erstmals) zur „Bemessung“ der Ansprüche gemäß \S 1615l, 1570 BGB nach „Höhe und Dauer“.

Danach trifft die Kindesmutter ab dem vollendeten dritten Lebensjahr des (jüngsten) Kindes grundsätzlich eine Erwerbspflicht. Weitergehende Ansprüche sind im Einzelfall bei „kindund elternbezogenen“ Gründen unter Billigkeitserwägungen zu prüfen, wobei die Gründe von der oder dem Unterhaltsberechtigten darzulegen und gegebenenfalls zu beweisen sind. ${ }^{6}$

Kindbezogene Gründe sind in diesem Zusammenhang (stets) vorrangig zu prüfen; ein „Altersphasenmodell, das bei der Frage der Verlängerung des Betreuungsunterhalts aus kindbezogenen Gründen allein oder wesentlich auf das Alter des Kindes abstellt, wird diesen Anforderungen nicht gerecht “. ${ }^{7}$ In dem Umfang, in dem das Kind nach Vollendung des dritten Lebensjahres eine kindgerechte Einrichtung besucht oder besuchen könnte, kann sich der betreuende Elternteil nicht mehr auf die Notwendigkeit einer persönlichen Betreuung des Kindes berufen. Dies gilt sowohl für den rein zeitlichen Aspekt der Betreuung als auch für den sachlichen Umfang der Betreuung in einer kindgerechten Einrichtung. ${ }^{8}$

Die Berücksichtigung elternbezogener Gründe ist hingegen Ausdruck der nachehelichen Solidarität, wobei die ausgeübte oder verlangte Erwerbstätigkeit des betreuenden Elternteils neben dem nach der Erziehung und Betreuung in einer Tageseinrichtung verbleibenden Anteil nicht zu einer „überobligatorischen " Belastung des betreuenden Elternteils führen darf. ${ }^{9}$

In der Praxis bedeuten die Entscheidungen des Bundesgerichtshofs, dass kindbezogene Gründe nur im Einzelfall vorliegen dürften, wie z.B. bei einer festgestellten geistigen oder körperlichen Behinderung oder gänzlich fehlender Betreuungsmöglichkeit. Problematischer einzuordnen sind „verhaltensauffällige“ Kinder, wie zum Beispiel solche mit ADHSSymptomen. Hier muss sich der betreuende Elternteil unter Umständen entgegenhalten lassen, dass eine Fremdbetreuung für das Kind sogar „,besser“ wäre.

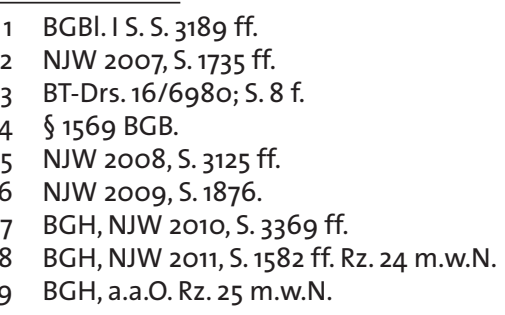


Elternbezogene Gründe sind demgegenüber - jedenfalls wenn ein Betreuungsangebot theoretisch zur Verfügung steht - mit Blick auf die Darlegungs- und Beweislast nicht erfolgversprechender, es sei denn, die Eheleute haben für die Zeit nach der Scheidung Vereinbarungen über den Unterhalt getroffen. ${ }^{10}$

Um den Aspekt der Vereinbarungen aufzugreifen: Diese werden regelmäßig im Rahmen eines Ehevertrages getroffen. ${ }^{11}$ Steht für die (künftigen) Ehegatten der Kinderwunsch schon fest, kann und sollte die Ehefrau darauf drängen, den Betreuungsunterhalt zu verlängern, um spätere Auseinandersetzungen über Erwerbsobliegenheiten zu vermeiden. Allerdings sollte nicht verkannt werden, dass „Mann“ dazu nur eingeschränkt bereit sein wird.

Der Vorteil einer Vereinbarung liegt - unabhängig von der Gestaltung im Einzelfall - zweifelsfrei in der Berechenbarkeit der Ansprüche im Fall einer Trennung.

\section{Unterhalt wegen Erwerbslosigkeit und Aufstockungsunterhalt} Voraussetzung für den Anspruch auf Aufstockungsunterhalt ist, dass die Unterhalt begehrende geschiedene Ehefrau oder der Unterhalt begehrende geschiedene Ehemann eine angemessene Erwerbstätigkeit ausübt oder sie bzw. ihn eine entsprechende Erwerbsobliegenheit trifft ${ }^{12}$; kann die oder der Berechtigte eine angemessene Tätigkeit ${ }^{13}$ nicht finden, folgt der Anspruch aus $\mathbb{} 1573$ Absatz 1 BGB.

Grundsätzlich ist der auf die Anspruchsgrundlagen entfallende Anteil durch das Gericht festzulegen, sofern nicht im Einzelfall darauf verzichtet werden kann. ${ }^{14}$

Die Praxis sieht von einer solchen Differenzierung häufig ab/hält sie nicht für notwendig. Auf eine Präzisierung sollte aber mit Blick auf eine spätere Abänderbarkeit der Ansprüche geachtet werden. Dies insbesondere, soweit auf Unterhalt gemäß $\int 1573$ Absatz 2 BGB (Aufstockungsunterhalt) erkannt worden ist.

Denn der Bundesgerichtshof hat für „Altfälle“ entschieden, dass bei einer (Erst-)Entscheidung nach dem 12. April $2006^{15}$ sich weder aus der anschließenden Senatsrechtsprechung noch aus dem Inkrafttreten des $\mathbb{S} 1578$ b BGB am 1. Januar 2008 eine wesentliche Änderung (der rechtlichen Verhältnisse) ergibt und auch $\mathbb{S} 36 \mathrm{Nr}$. 1 EGZPO in diesem Fall keine eigenständige Abänderungsmöglichkeit bietet. ${ }^{16}$

\section{Bestimmung des Bedarfs}

Das Unterhaltsrecht hat eine bedeutungsvolle Wendung durch die Rechtsprechung des Bundesgerichtshofs in Anwendung einer Vorschrift erfahren, die durch das Gesetz einer Neufassung in ihrem wesentlichen Grundgedanken - nämlich der Bestimmung des Unterhaltsbedarfs nach den ehelichen Lebensverhältnissen ${ }^{17}$ - unverändert geblieben ist.

So hat der Bundesgerichtshof in seiner Entscheidung vom 30. Juli 2008 judiziert, dass sich der nach den ehelichen Lebensverhältnissen zu bemessende Unterhaltsbedarf jeder und jedes Berechtigten - d.h. wenn beispielsweise der Unterhaltspflichtige sowohl der geschiedenen als auch der neuen Ehefrau Unterhalt schuldet - im Wege der Dreiteilung des Gesamtein- kommens der oder des Unterhaltspflichtigen und beider Unterhaltsberechtigten zu ermitteln ist. ${ }^{18}$

Von den Schwierigkeiten der Berechnung im Zusammenhang mit unterschiedlichen „Einsatzzeitpunkten“ einmal abgesehen, knüpft der Unterhaltsbedarf nicht nur an die wandelbaren ehelichen Lebensverhältnisse $\mathrm{an}^{19}$, sondern richtet sich - allein - nach der Lebensstellung der oder des Unterhaltspflichtigen und wird daraus abgeleitet. ${ }^{20}$

Bei konsequenter Anwendung dieser Prämisse hat danach die unterhaltsberechtigte - geschiedene - Ehefrau nicht nur die „neue“ Ehefrau hinzunehmen, sondern (auch) alle übrigen Unterhaltsberechtigten bis hin zu Adoptivkindern.

Die Rechtsprechung ist vielfach (kritisch) kommentiert

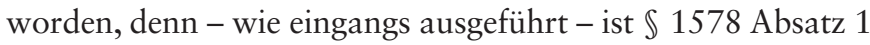
Satz 1 BGB in seiner Fassung unverändert.

In der Praxis war (und ist) die Drittelung kaum zu vermitteln, insbesondere nicht der geschiedenen Ehefrau, die nicht versteht, dass alle weiteren Unterhaltsberechtigten schon die ehelichen Lebensverhältnisse geprägt haben sollen. Die am Verfahren Beteiligten stoßen nicht selten bei der (gerichtlichen) Berechnung an ihre (Verständnis-)Grenzen, mit der Folge, dass eine Einigung kaum noch zu erzielen ist. Man kann es so formulieren, dass der „Methode“ im Ergebnis die Akzeptanz versagt geblieben ist.

Der Donnerhall vom Schlossplatz (Karlsruhe) in Gestalt des Beschlusses vom 25. Januar 2011 blieb nicht aus. ${ }^{21}$ Das Bundesverfassungsgericht hielt in seinem Leitsatz zur Entscheidung unter anderem fest, dass „unter Anwendung der Berechnungsmethode der so genannten Dreiteilung (sich die Rechtsprechung) von dem Konzept des Gesetzgebers zur Berechnung des nachehelichen Unterhalts (löst) und es durch ein eigenes Modell (ersetzt). Mit diesem Systemwechsel überschreitet sie die Grenzen richterlicher Rechtsfortbildung ...".

In der Anmerkung von Peter Gerhardt zu der Entscheidung ist vereinzelt nachzulesen, dass das Bundesverfassungsgericht die Berechnung (des BGH) nicht verstanden hat. ${ }^{22} \mathrm{Im}$ Übrigen merkt der Autor an, dass der Gesetzgeber (bewusst) keine „Festlegung getroffen (hat), ob neue nach der Scheidung entstandene Unterhaltslasten beim Bedarf oder bei der Leistungsfähigkeit zu berücksichtigen sind “ .23

Für die (Spruch-)Praxis ist damit nichts gewonnen. Denn die Entscheidung des Bundesverfassungsgerichts bleibt, auch wenn sie (richtigerweise) keine Vorgaben an den Gesetzgeber enthält, zu beachten. Inwieweit die Oberlandesgerichte dem

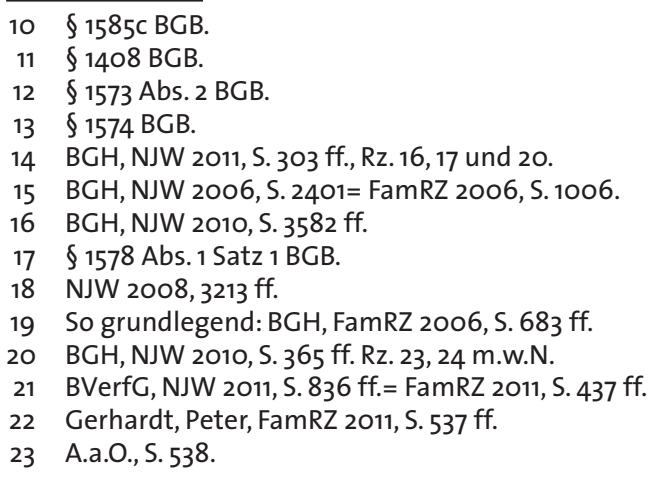


folgen werden oder aber die mahnenden Worte des Bundesgerichtshofs vernehmen, wonach die „Dreiteilung“ völlig unkompliziert zu handhaben und auch im Übrigen gesetzeskonform sei, bleibt abzuwarten.

Eine Frage ist damit jedoch nicht beantwortet: Auf welcher „Ebene“ sind die hinzugekommenen Unterhaltsberechtigten zu berücksichtigen? Prägen sie bereits den Bedarf nach den (ehelichen!) Lebensverhältnissen oder mindern sie die Leistungsfähigkeit der Unterhaltsschuldnerin oder des Unterhaltsschuldners?

\section{Herabsetzung und zeitliche Begrenzung des Unterhalts wegen Unbilligkeit}

Es darf eine vorsichtige Prognose dahin gewagt werden, dass keine Norm des Unterhaltsänderungsgesetzes nach Inkrafttreten so häufig Gegenstand von (veröffentlichten) Entscheidungen gewesen ist (und voraussichtlich auch bleibt) wie $\mathbb{} 1578 \mathrm{~b}$ BGB.

Vorauszuschicken ist auch hier, dass die Änderung der Rechtsprechung hinsichtlich der Gewichtung von Ehedauer und ehebedingten Nachteilen im Rahmen der Befristung bereits mit Urteil des Bundesgerichtsgehofs vom 12. April 2006 erfolgt ist und zwar unabhängig davon, ob aus der Ehe (auch) Kinder hervorgegangen sind, die von der oder dem Unterhaltsberechtigten betreut wurden. ${ }^{24}$ Davon ausgehend ist die oder der Unterhaltspflichtige mit dem Befristungseinwand präkludiert, wenn eine Entscheidung nach dem 12. April 2006 ergangen ist.

Ist Betreuungsunterhalt nach Vollendung des dritten Lebensjahres eines Kindes aus Billigkeitsgründen (noch) geschuldet, scheidet eine Befristung gemäß $\ 1578$ b BGB aus. ${ }^{25}$ Etwas anderes gilt für die Begrenzung, d.h. die oder der Unterhaltsberechtigte hat gegebenenfalls eine Kürzung auf den eigenen angemessenen Unterhalt hinzunehmen. ${ }^{26}$

Im Übrigen ist der nacheheliche Unterhalt zu begrenzen, wenn ein zeitlich unbegrenzter Anspruch unbillig wäre. ${ }^{27}$ Die einzelnen Billigkeitskriterien ergeben sich aus Satz 2 der genannten Bestimmung mit Verweis auf Absatz 1 Satz 2 und 3 (Herabsetzung).

Das Vorliegen ehebedingter Nachteile schränkt die Möglichkeit der Begrenzung und Befristung des Unterhalts regelmäßig ein. ${ }^{28}$ Derartige Nachteile können sich aus der Dauer der Pflege oder Erziehung eines gemeinsamen Kindes, aus der Gestaltung von Haushaltsführung und Erwerbstätigkeit während der Ehe sowie aus der Dauer der Ehe ergeben. ${ }^{29}$

Der angemessene Lebensbedarf im Sinne des $\mathbb{1 5 7 8 b}$ Absatz 1 BGB, der die Grenze für die Herabsetzung des nachehelichen Unterhalts bildet, bemisst sich nach der Rechtsprechung des XII. Senats nach dem Einkommen, welches die unterhaltsberechtigte Ehefrau oder der unterhaltsberechtigte Ehemann ohne die Ehe und Kindererziehung aus eigenen Einkünften zur Verfügung hätte. ${ }^{30}$

Erzielt die oder der Berechtigte Einkünfte, die den angemessenen Unterhaltsbedarf erreichen, kann dies im Rahmen der Billigkeitsabwägung nach einer Übergangszeit zum vollständi- gen Wegfall des nachehelichen Unterhalts in Form einer Befristung führen. Kann sie oder er diese Einkünfte nicht erreichen, scheidet eine Befristung des Anspruchs regelmäßig aus.

Nach einer „Übergangszeit" kann in diesen Fällen der Unterhalt bis auf den ehebedingten Nachteil herabgesetzt werden, der sich aus der „Differenz des angemessenen Unterhalts mit dem erzielten oder erzielbaren eigenen Einkommen ergibt, was (jedoch) voraussetzt, dass der eheangemessene Bedarf den angemessenen Lebensbedarf übersteigt" ".31

Um den ehebedingten Nachteil der Höhe nach bemessen zu können, bedarf es tatrichterlicher Feststellungen nicht nur zum angemessenen Unterhaltsbedarf, sondern auch zum Einkommen, das die oder der Unterhaltsberechtigte tatsächlich erzielt oder gemäß $\mathbb{S} 1574,1577$ BGB erzielen könnte ${ }^{32}$, das heißt es ist ein konkreter Sachvortrag zu einem nicht gelebten Leben notwendig.

Die Vielzahl der zur Herabsetzung und zeitlichen Begrenzung ergangenen Entscheidungen, insbesondere der Oberlandesgerichte, verdeutlicht, dass die Beratungspraxis vor einem nahezu unlösbaren Problem steht, nämlich der fehlenden Prognostizierbarkeit von Judikaten. Denn die Anwendung des $\$ 1578$ b BGB ist und bleibt - wie beschrieben - eine Frage des Einzelfalls.

\section{Fazit}

Ob der Gesetzgeber Handlungsbedarf sieht, halte ich für zweifelhaft, zumal das Bundesverfassungsgericht sich auf „Richterschelte" beschränkt hat. Das ist aus meiner Sicht aber auf die Zukunft gesehen kein Freibrief.

Gleichwohl hat der Blick realistisch zu bleiben und es ist mit einer Initiative aus dem Bundesministerium der Justiz zur „Reform der Reform“ in der gegenwärtigen - auch politischen - Situation nicht (ernsthaft) zu rechnen.

Dies schließt aber weder Initiativen von Verbänden noch eine breit gefächerte Diskussion aus.

In diesem Zusammenhang sollte auch über Alternativen zu Änderungen im materiellen Recht nachgedacht werden. Beispielhaft ist die Einführung einer Nichtzulassungsbeschwerde in Familienstreitsachen und Ehesachen zu nennen. Damit wäre auch der so häufig beschworene Gleichlauf der Verfahrensordnungen gewahrt. Kapazitäten des Bundesgerichtshofs stehen möglicherweise zur Verfügung, da beabsichtigt ist, $\mathbb{S} 7$ Insolvenzordnung aufzuheben. ${ }^{33}$ Dass der Freiraum bereits anderweitig verplant ist (Änderung des $\mathbb{5} 522 \mathrm{ZPO}$ ), sollte einer ergebnisoffenen Diskussion jedoch nicht im Weg stehen.

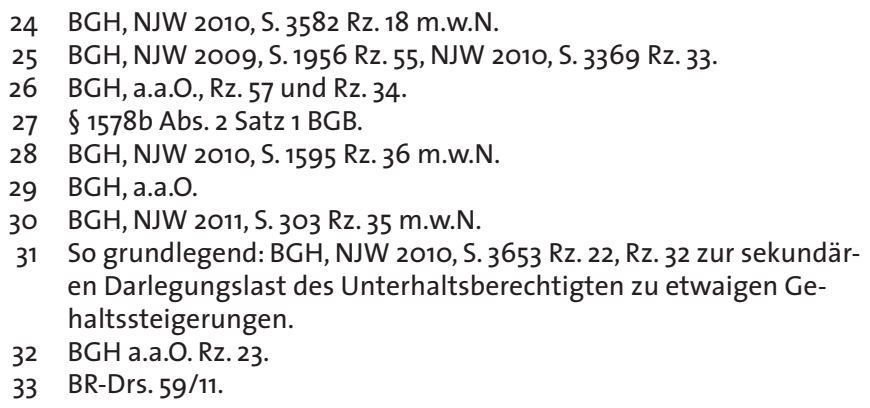

26 BGH, a.a.O., Rz. 57 und Rz. 34.

$27 \S 1578$ b Abs. 2 Satz 1 BGB.

28 BGH, NJW 2010, S. 1595 Rz. 36 m.w.N. en Darlegungslast des Unterhaltsberechtigten zu etwaigen $\mathrm{Ge}$ haltssteigerungen.

33 BR-Drs. 59/11. 